\title{
Evolutionary stasis of viruses?
}

\section{Edward C. Holmes (10) and Sebastián Duchêne}

In their recent Opinion article (Prisoners of war - host adaptation and its constraints on virus evolution. Nat. Rev. Microbiol. https:// doi.org/10.1038/s41579-018-0120-2 (2018))1, Simmonds et al. use studies of hepatitis B virus $(\mathrm{HBV})^{2}$ and $\mathrm{B} 19$ parvovirus ${ }^{3}$ from Bronze Age remains, along with endogenous viruses ${ }^{4}$, to propose that evolutionary rates in viruses decline massively as they adapt to hosts. Although these data have changed our perspective on evolutionary timescales, we disagree that they predict long-term evolutionary stasis.

Simmonds et al. claim that there is "increasing evidence for extreme genetic conservation of viruses over longer periods of evolution". However, most families and genera of RNA viruses exhibit limited sequence similarity $^{5}$, and as expected with rapid evolution over long time periods, even the amino acid sequences of the most conserved proteins can be difficult to align ${ }^{5}$. We believe that the regression analysis of rates of viral evolution performed by Simmonds et al. is misleading as it combines single host associations with multi-host comparisons. Importantly, the deeper the timescale of analysis the more virus-host associations are compared, such that the lowest rate estimates coincide with the highest frequency of host-jumping, counter to their adaptive model.

Isolates of $\mathrm{HBV}$ dating back to the sixteenth century lack temporal structure ${ }^{6}$. Accurately estimating an evolutionary rate therefore requires a longer sampling period ${ }^{6}$, as confirmed by the Bronze Age samples ${ }^{2}$. Hence, higher rate estimates for $\mathrm{HBV}$ are erroneous and likely reflect counts of transient mutations, as expected under time-dependent virus evolution ${ }^{7,8}$. Although there is temporal structure among recent $\mathrm{B} 19$ isolates ${ }^{9,10}$, this may again reflect time dependence or the impact of a small number of ancient samples in the regression. Simmonds et al. also claim that our earlier paper ${ }^{10}$ "predicted a time of origin of current genotype 1 strains to the 1960 s or 1970 s". However, no divergence times were presented in this paper, but were previously by Simmonds and colleagues ${ }^{9}$. Moreover, no data are presented for the lowest rate of spumavirus evolution covering $\sim 750$ million years. The relatively slow evolution of spumaviruses may reflect low rates of replication ${ }^{11}$ and the occurrence of nonsynonymous substitutions argues against extreme purifying selection ${ }^{11}$.
We believe that there is no biological reason why the evolutionary rate in RNA viruses, which encode their own RNA polymerase, should decline to that of hosts that use entirely different replication enzymes. The likely changing nature of the complex environments faced by viruses, combined with the size of sequence space $\left(\sim 4^{10,000}\right.$ for a typical RNA virus), make evolutionary stasis unrealistic. For example, although influenza viruses have probably been associated with wild bird species for millennia, their evolutionary rates are of the same magnitude as the mammals in which they periodically emerge ${ }^{12,13}$. Finally, the high mutation rates in RNA viruses ensure that evolutionary stasis would result in massive purifying selection and an enormous mutational load.

Viruses are ancient entities and their remarkable sequence diversity reflects a long evolutionary history characterized by high rates of genetic change. Along with host adaptation, much of the apparent discrepancy between short-term and long-term evolution in viruses may be an illusion caused by the inappropriate use of molecular clock dating without temporal structure, incorrect calibration points, differences in replication rates, site saturation that is even apparent at shallow genetic distances ${ }^{14}$ and the inherent time dependence of evolutionary rates ${ }^{8}$.

There is a reply to this letter by Simmonds, $\mathrm{P}$., Aiewsakun, P. \& Katzourakis, A. Nat. Rev. Microbiol. https://doi.org/10.1038/s41579019-0169-6 (2019).
Edward C. Holmes (iD ${ }^{1 *}$ and Sebastián Duchêne ${ }^{2}$ ${ }^{\prime}$ Marie Bashir Institute for Infectious Diseases and Biosecurity, Charles Perkins Centre, School of Life and Environmental Sciences and Sydney Medical School, The University of Sydney, Sydney, Australia. 2 Department of Biochemistry and Molecular Biology, Bio21 Molecular Science and Biotechnology Institute, University of Melbourne, Melbourne, Australia. *e-mail: edward.holmes@sydney.edu.au https://oi.org/10.1038/s41579-019-0168-7

1. Simmonds, P., Aiewsakun, P. \& Katzourakis, A. Prisoners of war - host adaptation and its constraints on virus evolution. Nat. Rev. Microbiol. https://doi. org/10.1038/s41579-018-0120-2 (2018).

2. Muhlemann, B. et al. Ancient hepatitis B viruses from the bronze age to the medieval period. Nature 557, 418-423 (2018)

3. Mühlemann, B. et al. Ancient human parvovirus B19 in Eurasia reveals its long-term association with humans. Proc. Natl Acad. Sci. USA 115, 7557-7562 (2018).

4. Katzourakis, A. \& Gifford, R. J. Endogenous viral elements in animal genomes. PLOS Genet. 6 , e1001191 (2010).

5. Shi, M. et al. Redefining the invertebrate virosphere. Nature 540, 539-543 (2016).

6. Patterson Ross, Z. et al. The paradox of HBV evolution as revealed from a 16 th century mummy. PLOS Pathog. 14, e1006750 (2018).

7. Duchêne, S. et al. Analyses of evolutionary dynamics in viruses are hindered by a time-dependent bias in rate estimates. Proc. R. Soc. B 281, 20140732 (2014).

8. Ho, S. Y. et al. Time-dependent rates of molecular evolution. Mol. Ecol. 20, 3087-3101 (2011).

9. Norja, P. et al. Rapid sequence change and geographical spread of human parvovirus B19: comparison of B19 virus evolution in acute and persistent infections. J. Virol 82 6427-6433 (2008)

10. Shackelton, L. A. \& Holmes, E. C. Phylogenetic evidence for the rapid evolution of human B19 erythrovirus. J. Virol. 80, 3666-3669 (2006)

11. Switzer, W. M. et al. Ancient co-speciation of simian foamy viruses and primates. Nature $434,376-380$ (2005).

12. Fourment, M. \& Holmes, E. C. Avian influenza virus exhibits distinct evolutionary dynamics in poultry and wild birds. BMC Evol. Biol. 15, 120 (2015).

13. Worobey, M. et al. A synchronized global sweep of the internal genes of modern avian influenza virus. Nature 508, 254-257 (2014)

14. Duchêne, $\mathrm{S}$. et al. Declining transition/transversion ratios through time reveal limitations to the accuracy of nucleotide substitution models. BMC Evol. Biol. 15 , 36 (2015).

Competing interests

The authors declare no competing interests.

\section{Reply to 'Evolutionary stasis of viruses?'}

\section{Peter Simmonds, Pakorn Aiewsakun and Aris Katzourakis}

Our Opinion article (Prisoners of war host adaptation and its constraints on virus evolution. Nat. Rev. Microbiol. https://doi. org/10.1038/s41579-018-0120-2 (2018)) ${ }^{1}$ describes host adaptation and its potential role in shaping long-term evolution of viruses. Holmes and Duchêne's correspondence (Evolutionary stasis of viruses? Nat. Rev. Microbiol. https://doi.org/10.1038/s41579-0190168-7 (2019)) $)^{2}$ closely concurs that viruses are fast-evolving and diverse ancient entities, constrained by host adaptation and that various methodological artefacts can influence rate measurements. The more than 1 millionfold range in evolutionary rates observed in viruses far exceeds those typically observed in cellular genes ${ }^{3}$. This contrast prompted us to advance the perspective that the adaptability of viruses, intensity of fitness selection and large effective population sizes drive sequence change in a quantitatively different way from macroorganisms. Including the host in our model places unfamiliar constraints on viruscentric accounts of evolution, where over the 